\title{
Erratum: Unusual marine unicellular symbiosis with the nitrogen-fixing cyanobacterium UCYN-A
}

Jonathan P. Zehr, Irina N. Shilova, Hanna M. Farnelid, Maria del Carmen Muñoz-Marín and Kendra A. Turk-Kubo

Nature Microbiology 2, 16214 (2016); published 20 December 2016; corrected 23 January 2017

In the original version of this Review article, co-author Maria del Carmen Muñoz-Marín's name was coded wrongly, resulting in it being incorrect in the HTML version of the article and when exported to citation databases. This has now been corrected. 